\title{
Caso clínico: Manifestación Extrapulmonar de Neoplasia Indiferenciada.
}

\section{*Correspondencia:}

drjorgetomala@gmail.com

Teléfono [593] 992491338

Conflicto de intereses: Los autores declaran no tener conflictos de intereses.

Fondos: Ver la página 167

Recibido: 1 Marzo 2018 Aceptado: 11 Julio 2018

Publicado: 30 Agosto 2018

Membrete bibliográfico:

Tomalá J, Loero G. Caso clínico: Manifestación Extrapulmonar de Neoplasia Indiferenciada. Rev. Oncol. Ecu 2018;28(2):163-168.

DOI: https://doi.org/10.33821/261

Copyright Tomalá, et al. Este artículo es distribuido bajo los términos de Creative Commons Attribution License, el cual permite el uso y redistribución citando la fuente $y$ al autor original.

\section{Clinical case: Extrapulmonary Manifestation of Undifferentiated Neoplasia.}

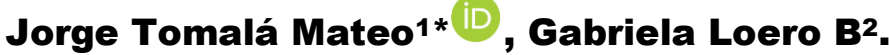

1. Postgrado de Medicina Interna de la Universidad de Guayaquil- Ecuador.

2. Instituto Oncológico Nacional “Dr. Juan Tanca Marengo”, Solca-Guayaquil.

\section{Resumen}

Introducción: El síndrome de neoplasia primaria de origen desconocido, se refiere a la presencia de lesiones metastásicas corroboradas histológicamente cuyo origen primario no puede ser identificado durante la evaluación pre-tratamiento. En el presente caso clínico se presenta una manifestación extrapulmonar de neoplasia no diferenciada por su rareza y descripción del estudio diagnóstico.

Caso Clínico: Se trata de un hombre de 49 años, con una masa axilar derecha de 1 año de evolución, de 30 x $20 \mathrm{~cm}$, que incluye el hemitórax, asociado a dolor 7/10, con valoración de la escala Eastern Cooperative Oncology Group (PS ECOG) de 3 en forma inicial. Fue tratado por sepsis por infección de lecho tumoral. La tomografía reportó la lesión tumoral extensa en región axilar derecha con áreas de necrosis, burbujas aéreas, una lesión nodular subcentimétrica en segmento apical de lóbulo superior del pulmón derecho y derrame pleural. La biopsia en cuña reportó una Neoplasia maligna indiferenciada CD-56 +, CD-79 A +, VIMENTINA +, CAM $5.2+$, CK - $20+$ +Se concluyó como manifestación extrapulmonar metastásica de neoplasia maligna indiferenciada de origen pulmonar posiblemente de origen neuroendócrino.

Evolución: Se estableció un tratamiento neoadyuvante con cisplatino + etopósido, por 3 meses. Escala PS ECOG 1, recuperación funcional de la extremidad, reducción tumoral del 50 \% de lesión tumoral, en espera de tratamiento quirúrgico.

Conclusión: En este reporte se presenta un caso de síndrome de metástasis de primario oculto el cual responde con reducción tumoral al tratamiento Neoadyuvante. Aunque son de mal pronóstico en la mayoría de los pacientes, en este reporte se presenta una respuesta adecuada al tratamiento.

Palabras Claves: METÁSTASIS DE LA NEOPLASIA, NEOPLASIAS.

DOI: $10.33821 / 261$ 


\section{Abstract}

Introduction: Primary neoplasia syndrome of unknown origin refers to the presence of histologically corroborated metastatic lesions whose primary origin can not be identified during the pre-treatment evaluation. In the present clinical case an extrapulmonary manifestation of undifferentiated neoplasia is presented due to its rarity and description of the diagnostic study.

Clinical Case: This is a 49-year-old man, with a right axillary mass of 1 year evolution, $30 \times 20 \mathrm{~cm}$, which includes the hemithorax, associated with pain 7/10, with assessment of the Eastern Cooperative Oncology Group scale (PS ECOG) of 3 in initial form. He was treated for sepsis due to infection of the tumor bed. The tomography reported the extensive tumor in the right axillary region with areas of necrosis, air bubbles, a subcentimeter nodular lesion in the apical segment of the upper lobe of the right lung and pleural effusion. The wedge biopsy reported an undifferentiated malignancy of CD-56 +, CD-79 A +, VIMENTINA +, CAM 5.2 +, CK - $20+$. It was concluded as a metastatic extrapulmonary manifestation of undifferentiated malignancy of pulmonary origin possibly of neuroendocrine origin.

Evolution: A neoadjuvant treatment with cisplatin + etoposide was established for 3 months. PS ECOG 1 scale, functional recovery of the limb, tumor reduction of $50 \%$ of tumor lesion, awaiting surgical treatment.

Conclusion: This report presents a case of metastasis syndrome of the occult primary which responds with tumor reduction to Neoadjuvant treatment. Although they are of poor prognosis in most patients, this report presents an adequate response to treatment.

Keywords: NEOPLASMS METASTASIS, NEOPLASMS.

DOI: $10.33821 / 261$

\section{Introducción}

El síndrome de neoplasia primaria de origen desconocido, se refiere a la presencia de lesiones metastásicas corroboradas histológicamente cuyo origen primario no puede ser identificado durante la evaluación pre-tratamiento. Constituye hasta un $5 \%$ de todos los casos de cáncer y son considerados de mal pronóstico con una sobrevida global de hasta 9 meses. El sitio primario es hallado en menos del $30 \%$ de los casos.

Los sitios afectados con mayor frecuencia son: ganglios linfáticos, hígado, pulmón y hueso. La presentación clínica es muy variable dependiendo la localización metastásica. La PETTC es la técnica de imágenes de elección en el estudio pre-tratamiento. La biopsia en cuña es la técnica de elección para diagnóstico patológico con técnica de inmunohistoquímica que nos orientará en la identificación del tumor primario [1-3]. En el presente caso clínico se presenta una manifestación extrapulmonar de neoplasia no diferenciada por su rareza y descripción del estudio diagnóstico. 


\section{Caso Clínico}

Paciente masculino de 49 años de edad, sin antecedentes patológicos conocidos, con masa axilar derecha de 1 año de evolución, de aproximadamente $30 \times 20 \mathrm{~cm}$ (figura 1), que incluye el hemitórax derecho hasta $1 / 3$ por encima de flexura del codo ipsilateral, con signos de congestión venosa y linfática, asociado a dolor con una escala de valoración visual analógica (VAS) de 7/10, con valoración de la escala Eastern Cooperative Oncology Group (PS ECOG) de 3 en forma inicial.

Intercurrió con sepsis severa por infección de lecho tumoral. Se realizan estudios de estadificación con una Tomografía Corporal Total, la cual reportó una lesión tumoral extensa en región axilar derecha de $27 \times 17 \mathrm{~cm}$, heterogénea con áreas de necrosis y burbujas aéreas, lesión nodular subcentimétrica en segmento apical de lóbulo superior del pulmón derecho y derrame pleural bilateral. Una vez resuelto el cuadro séptico se realizó biopsia en cuña de masa axilar derecha, con reporte patológico de Neoplasia maligna indiferenciada CD - $56+$, CD - 79 A +, VIMENTINA +, CAM 5.2 +, CK - $20+$. Se realizó una Endoscopía Digestiva Alta y Video Colonoscopía las cuales fueron normales. Posterior a discusión de caso se concluyó como manifestación extrapulmonar metastásica de neoplasia maligna indiferenciada de origen pulmonar posiblemente de origen neuroendócrino.

Figura 1. Fotografías y tomografías de la masa Tumoral

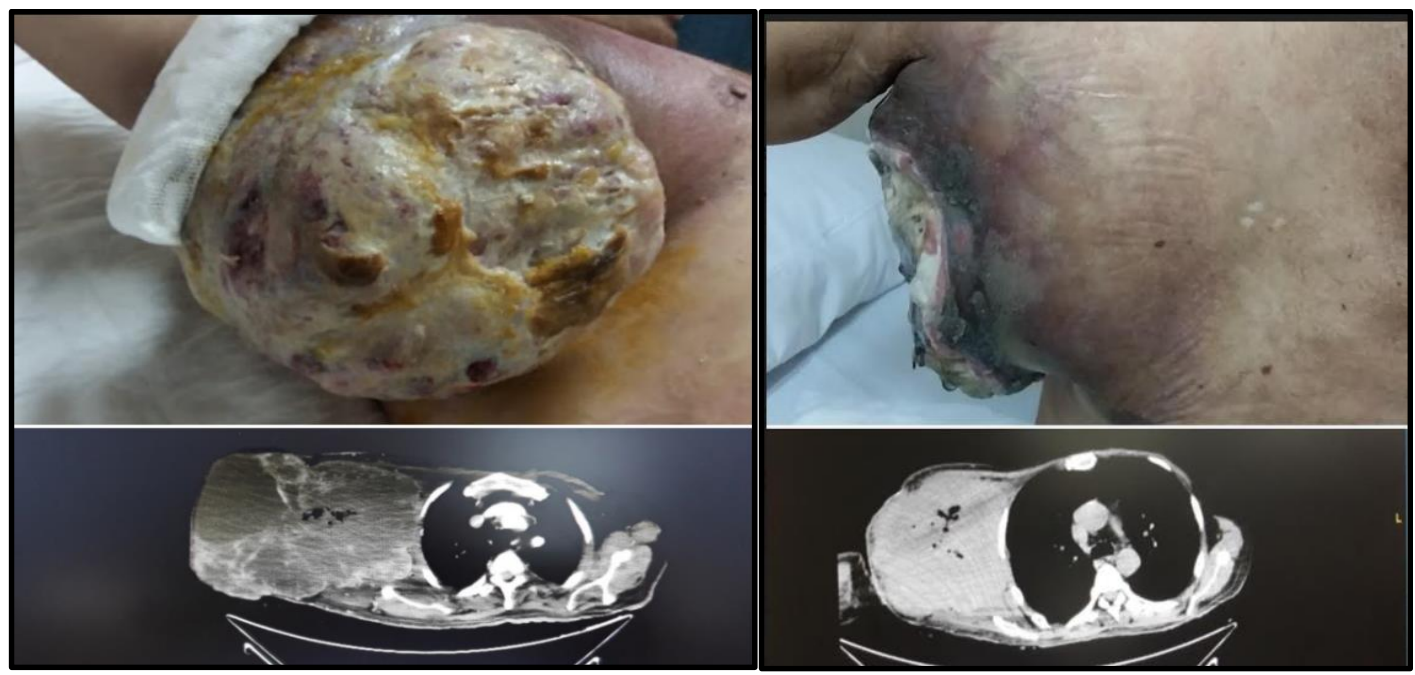

Imágenes superiores demuestran masas tumoral axilar. Imágenes inferiores cortes axiales del tórax en donde se describe una masa sólida extrapulmonar derecha.

\section{Evolución}

Se estableció un tratamiento neoadyuvante con cisplatino + etopósido, el cual se realizó durante 3 meses, con toxicidad hematológica (neutropenia) superada con Estimulantes de 
la colonia de Granulocitos. Actualmente paciente con PS ECOG 1, recupera funcionalidad de miembro superior derecho y reducción clínica del 50\% de lesión tumoral, sin signos de congestión linfovascular. Paciente en espera de tratamiento quirúrgico por buena tasa de respuesta a la inducción.

\section{Discusión}

El síndrome de metástasis de primario desconocido, constituye 4 entidades bien características: el Adenocarcinoma bien diferenciado (60\%), el Carcinoma indiferenciado (30\%), el Carcinoma de células escamosas (5\%) y la Neoplasia maligna pobremente diferenciada (5\%).

En este contexto el aspecto al microscopio óptico no permite clasificarlo dentro de ninguna categoría neoplásica general, ya que deben ser identificados con técnicas de Inmuno Histo Química (IHQ) por un patólogo experto.

Los diagnósticos más frecuentes incluyen linfoma no hodgkin, sarcoma, melanoma y tumores neuroendócrinos indiferenciados. Existe un subgrupo de neoplasias indiferenciadas (carcinomas neuroendócrinos de células pequeñas con manifestaciones extrapulmonares) que afecta a ganglios linfáticos cervicales, axilares y retroperitoneales, es de buen pronóstico y presenta gran respuesta a la quimioterapia con sales de platino y etopósido.

En este caso clínico los hallazgos IHQ fueron controversiales, ya que la positividad para CD 79 A, orientan a neoplasia de células plasmáticas, y por otro lado CK $7(-)$, CK 20 (+) y CAM $5.2(+)$ apoyan el diagnóstico de carcinoma colónico, apendicular, vejiga y estómago [2].

\section{Conclusiones}

En este reporte se presenta un caso de síndrome de metástasis de primario oculto el cual responde con reducción tumoral al tratamiento Neoadyuvante. Aunque son de mal pronóstico en la mayoría de los pacientes, en este reporte se presenta una respuesta adecuada al tratamiento.

\section{Agradecimientos}

Un agradecimiento al paciente que autorizó la publicación el presente caso. 


\section{Información adicional}

Nota del Editor

La Revista Oncología Ecu permanece neutral con respecto a los reclamos jurisdiccionales en mapas publicados y afiliaciones institucionales.
Abreviaturas

IHQ: Inmuno Histo-química.

PS ECOG: Eastern Cooperative Oncology Group.

VAS: Escala Visual Analógica.

VCC: Video Colonoscopía.

VEDA: Video Endoscopía Digestiva Alta

Archivos Adicionales

Ninguno declarado por los autores.

Fondos

Los fondos de la investigación fueron propios de los autores del presente artículo.

\section{Disponibilidad de datos y materiales}

Existe la disponibilidad de datos bajo solicitud al autor de correspondencia. No se reportan otros materiales.

\section{Contribuciones de los autores}

JTM realizó la idea de investigación, revisión bibliográfica. GLB recolección de datos, escritura del artículo. JTM realizó el análisis crítico del artículo y las correcciones editoriales. Todos los autores leyeron y aprobaron la versión final del artículo.

Aprobación de ética y consentimiento para participar

No aplica a este estudio.

\section{Consentimiento para publicación}

Los autores cuentan con el permiso escrito de la paciente para publicar el caso clínico. 
Información de los autores

Jorge Tomalá Mateo, Médico Internista. Postgradista de Medicina Interna, SOLCA Guayaquil, período 2008 - 2011 (iD https://orcid.org/0000-0003-2967-8731

Gabriela Loero B, Médico del Instituto Oncológico Nacional "Dr. Juan Tanca Marengo" Solca-Guayaquil.

\section{Referencias}

Abreviaturas en la referencias

DOI: Digital Object

Identifier

PMID: PubMed Identifier

SU: Short URL
1. Fizazi K, Greco FA, Pavlidis N, Daugaard G, Oien K, Pentheroudakis G. Cancers of unknown primary site: ESMO Clinical. Ann Oncol 2015;26(suppl 5):133-138.

2. Losa F, Soler G, Casado A, Estival A, Fernández I, Giménez S,et al. SEOM clinical guideline on unknown primary cancer (2017). Clin Transl Oncol. 2018;20(1):89-96. DOI: 10.1007/s12094-017-1807-y.

3. Tamam MO, Mulazimoglu M, Guveli TK, Tamam C, Eker O, Ozpacaci T. Prediction of survival and evaluation of diagnostic accuracy whole body 18F-fluoro-2-deoxyglucose positron emission tomography/computed tomography in the detection carcinoma of unknown primary origin. Eur Rev Med Pharmacol Sci. 2012;16(15):2120-30. PubMed PMID: 23280029. 